\title{
Health promotion strategies for the prevention and control of non- communicable diseases in Nigeria
}

\author{
Article by Okafor $\mathrm{CN}^{1}$, Young $\mathrm{EE}^{2}$, Nwobi $\mathrm{AE}^{3}$ \\ ${ }^{1}$ Department of Physiology, University of Nigeria Enugu Campus \\ ${ }^{2}$ Department of Medicine University of Nigeria Enugu Campus \\ ${ }^{3}$ Department of Community Medicine, University of Nigeria, \\ Enugu Campus. \\ Email:-chinyere.okafor@unn.edu.ng,ekenechukwu.young@unn.edu.ng, \\ amaebie@yahoo.com.
}

\begin{abstract}
Non-communicable diseases have shown an increasing impact on health status of populations globally with a rising rates in developing countries especially Nigeria. However, government in Nigeria are not keeping pace with the ever expanding needs for policies, legislative services and infrastructure to prevent NCDs. This paper is aimed at identifying the modifiable risk factors for NCD in Nigeria, the determinants of health involved and the health promotion strategies which can be initiated and implemented at various sectors. Secondary data was obtained from results of search from WHO NCD country profiles 2014, WHO global status report on NCD 2011, WHO global action plan for prevention and control of NCD 2013-2020 and Health Reform Foundation of Nigeria 2011 amongst others. The results showed that the modifiable risk factors included tobacco smoking, alcohol consumption, raised blood pressure and obesity with about 6\%,10\%,35\% and 6.5\% respectively. The determinants of health spanned from economic, natural and social environments amongst sustainability, transport, lifestyle and community services. Health education, nutritional intervention, lifestyle and behavioral changes in addition to environmental modifications are strategies which can help prevent and control NCD through addressing the risk factors and determinants.
\end{abstract}

Keywords: Non-communicable diseases, Health Promotion

\section{Introduction}

The increasing rise in the prevalence of Non-Communicable Diseases (NCDs) has continued unabated in Nigeria and in other regions of the world; as NCDs have constituted about 24\% of deaths in Nigeria [1].The WHO 2011 report projects that NCDs will be the leading cause of deaths globally exceeding communicable, maternal, perinatal and nutritional disease by 2030. The rise in the burden of NCDs in low and middle income countries has been attributed to increasing levels of the risk factors which are linked to adoption of western culture and values [2]. Despite the increasing prevalence of risk factors for NCDs, most developing countries including Nigeria have no current and reliable population based data on the burden of these risk factors to guide programs targeted at control [3].

\section{Understanding of concepts}

Non-communicable diseases (NCDs) are diseases with complex etiology, multiple risk factors, and long latency period often leaving a functional impairment or disability which are in most cases incurable but can only be controlled. They include the "big four"- cardiovascular disease, cancer, diabetes, and chronic obstructive pulmonary disease, among others.

Risk factors are conditions, which predispose an individual to ill health. The prevalence of the risk factors for NCDs varies between income groups and differs with gender [4]. They can be divided into modifiable and non-modifiable risk factors. 
The modifiable risk factors are those that can be prevented and controlled e.g. environmental risks and lifestyle, which can further be divided into metabolic i.e., dyslipidemia, elevated glucose, raised blood pressure, overweight and obesity, and behavioral risk factors i.e. physical inactivity, tobacco use, alcohol use, unhealthy diets i.e. increased fat and sodium, low intake of fruits and vegetables. The nonmodifiable risk factors cannot be prevented or controlled, they are in-born e.g. age, gender, genetic factors, race.

Health Promotion is the processes of enabling people increase control over their health and improve it [5]. It involves a combination of health, education, economic, political, spiritual or organizational initiatives designed to bring about positive attitudinal, behavioral, social or environmental changes conducive for improving the health of populations.

The key to controlling the rising prevalence of NCDs in the world is primary prevention, which is aimed at eradicating, eliminating or minimizing the impact of disease through comprehensive population based programs. The WHO approaches for primary prevention includes population (mass) strategy and high-risk strategy [6].To do this identification of major risk factors as well as their prevention and control is necessary. Although NCDs have become prevalent globally, the use of health promoting services, which are necessary have been inadequate in many countries including Nigeria. Till date, there has been limited focus on these issues in the health policy. The prime attention needed is to adopt a process, which addresses not only individual unhealthy behavior but also determinants of health to promote and sustain good health. The aim is to identify the health promotion strategies for the prevention and control of NCDs in Nigeria. This will be achieved by identifying the modifiable risk factors for NCDs and the determinants of health involved. In addition we will aim to elaborate means of creating health promotion strategies for the prevention and control of NCDs.

\section{Literature review}

The World Health Organization (WHO) Global Status Report on Noncommunicable Diseases (NCDs) 2010 showed that NCDs are the highest cause of death worldwide [7]. According to WHO 2011 report, NCDs were responsible for $63 \%$ of all deaths globally in 2008 (about 36 million people), mainly from cardiovascular diseases (48\%), cancers (21\%), chronic respiratory diseases (12\%), and diabetes (3\%) with more than $80 \%$ occurring in developing countries [8]. According to WHO 2010 report, more than nine million of these deaths occurred before the age of 60 and could have largely been prevented. WHO 2004 report shows that NCDs are responsible for 3 times as many disability adjusted life years. Premature deaths from NCDs range from 22\% among men to $35 \%$ among women in low-income countries and were largely attributed to poor diet, insufficient physical activity, tobacco use, and harmful use of alcohol [1].

According to the WHO 2014 report, in Nigeria death from NCD accounts for 24\% of total deaths [1]. Probability of dying between 30-70years from NCD is 20\% [1]. Death is projected to rise to about 5 million by 2015, with $52 \%$ from diabetes. The economic cost of NCD in Nigeria in 2005 was about \$400million USD from premature death due to NCD and it is estimated to rise to about \$8billion USD by 2015 [9].The loss from these diseases affect not only the individual but greatly impact on the nation`s output. In Nigeria, only about $49.6 \%$ live in urban community [1] and the risk factors are as shown in the figure below.

According to the WHO draft of global action plan for the prevention and control of NCDs 2013-2020 [10], the vision was to create a world free of avoidable burden of NCDs and its goal was to reduce the preventable and avoidable burden of morbidity, mortality and disability due to NCDs by means of multi-sectorial collaboration and 
co-operation at national, regional and global levels, so that populations will reach the highest attainable standards of health and productivity at every age and those diseases will no longer be a barrier to well-being or socioeconomic development of the people. Its targets include a $25 \%$ relative reduction in risk of premature mortality from cardiovascular diseases, cancer, diabetes, or chronic respiratory diseases, at least $10 \%$ relative reduction in the harmful use of alcohol, as appropriate, within the national context, a $10 \%$ relative reduction in prevalence of insufficient physical activity, a $30 \%$ relative reduction in mean population intake of salt/sodium, a 30\% relative reduction in prevalence of current tobacco use in persons aged 15+ years, a $25 \%$ relative reduction in the prevalence of raised blood pressure or contain the prevalence of raised blood pressure, according to national circumstances, to halt the rise in diabetes and obesity, at least $50 \%$ of eligible people receive drug therapy and counselling (including glycemic control) to prevent heart attacks and strokes among others.

\section{Methodology}

Information was obtained using PubMed search, Google and Google Scholar to extract resources on NCDs globally, in developing countries and in Nigeria. These included prevalence studies and systematic reviews. Search terms included a combination of; non-communicable disease, cardiovascular disease, diabetes mellitus, cancer, chronic obstructive pulmonary disease, physical activity among others. Other sources of information were from WHO reports, while various study modules and textbooks were used as foundation text.

\section{Discussion}

\section{Non-communicable diseases and their modifiable risk factors}

Majority of NCDs occur in individuals with modest elevations of multiple risk factors than a single risk factor and they often co-exist. They are chronic in nature.

Cardiovascular diseases (CVD) are diseases that involve the heart and the blood vessels. They include ischemic heart diseases (IHD), stroke, hypertensive heart disease etc. The basic mechanism of disease is mostly through atherosclerosis, which usually occurs following presence of risk factors like high blood pressure, smoking, lack of exercise, obesity among others. CVDs are the leading cause of death globally except in Africa [11] though the prevalence has been increasing following adoption of western culture and habits. About 17.3 million deaths were recorded in 2013 from CVDs [12] with IHD and stroke accounting for $80 \%$ and $75 \%$ deaths in males and females respectively [11].In Nigeria, CVD accounted for 145,810 deaths (7\%) in 2013 [1].

Diabetes mellitus is a metabolic disease which presents with a high blood glucose level with symptoms of frequent urination, increased thirst and hunger. There are 4 main types, Type 1, Type 2, gestational diabetes and pre-diabetes. Complications can be acute or chronic. The chronic complications include CVDs, stroke and damage to the eyes. The risk factors especially Type 2 is unhealthy diet, lack of exercise, obesity etc. In 2014, about 387 million people were recorded to have diabetes mellitus worldwide [13] with most cases presenting with Type 2 [14] and the prevalence is estimated to rise to 592 million by 2035 [13].The global economic cost of diabetes in 2014 was estimated at $\$ 612$ billion USD [15].In Nigeria, diabetes accounted for 41,660 deaths (2\%) in 2013 [1].

Cancer or malignant tumor or neoplasm, is a chronic debilitating disease involving abnormal cell growth that can spread to other parts of the body. Most cancers occur following exposure to risk factors. E.g. tobacco use causes about $22 \%$ of deaths from cancer [16], $10 \%$ are from obesity, lack of exercise, poor diet and consumption of alcohol [16]. Other factors are environmental pollutants, exposure to ionizing radiation etc. In 2012, 14.1 million new cases of cancer were recorded globally 
South American Journal of Public Health

Volume 4, Issue 1, 2016

excluding skin cancer other than melanoma [16].It was responsible about 8.2 million deaths [17]with rates increasing in the developing world from lifestyle changes and longer life expectancy [18].

In Nigeria, Cancer accounted for 62,490 deaths (3\%) in 2013 [1].

Chronic obstructive pulmonary disease (COPD) is a lung disease characterized by chronic poor airflow [19]. The most common cause of COPD is tobacco smoking, with air pollution and genetic factors playing a lesser role [20].In the developing world, one of the common sources of air pollution is from vented cooking and heating fires. Second hand smoke is also a risk factor. Globally, COPD affects 329 million people [21] and in 2013, 2.9 million deaths were caused by COPD [22]. In Nigeria, COPD accounted for 20,830 deaths (1\%) in 2013 [1].

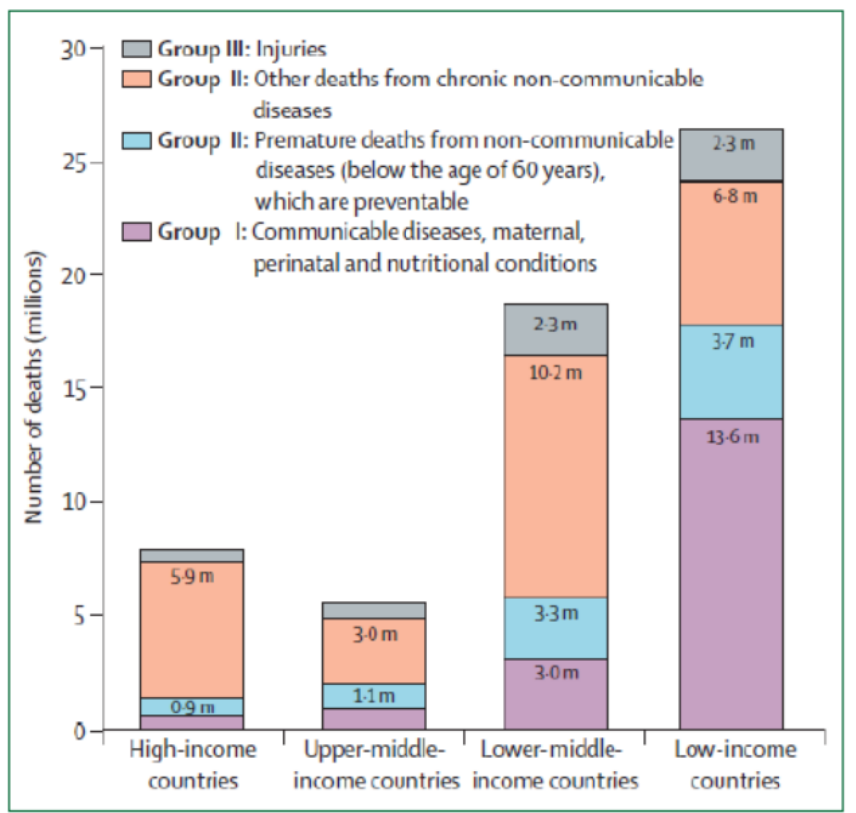

Figure 1. Total number of deaths in the world in 2004 (Geneau et al, 2010) Source: NCD Alliance, Nutrition, physical activity and NCD prevention working group. 


\section{Nigeria}

Total population: 169000000 Income Group: Lower middle

Age-standardized death rates*

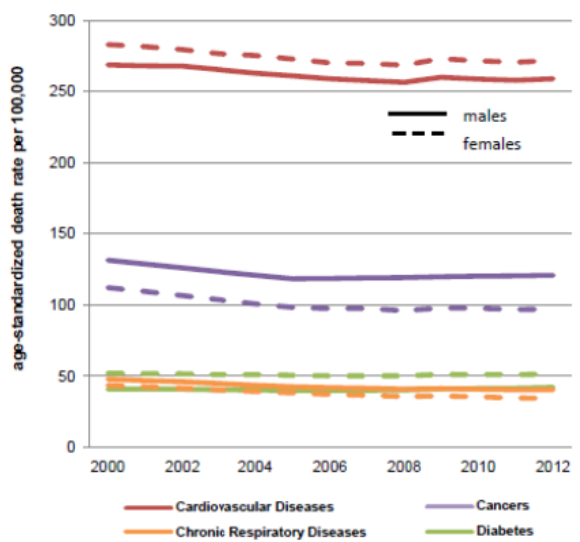
Population proportion between ages 30 and 70 years: $27.7 \%$

Proportional mortality (\% of total deaths, all ages, both sexes) ${ }^{*}$

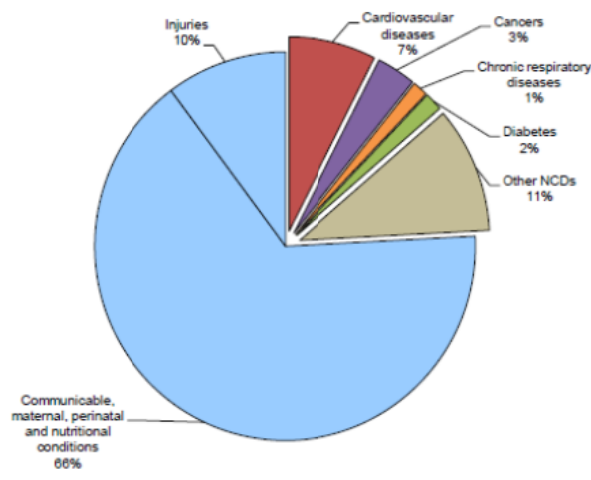

Total deaths: $2,083,000$

NCDs are estimated to account for $24 \%$ of total deaths.

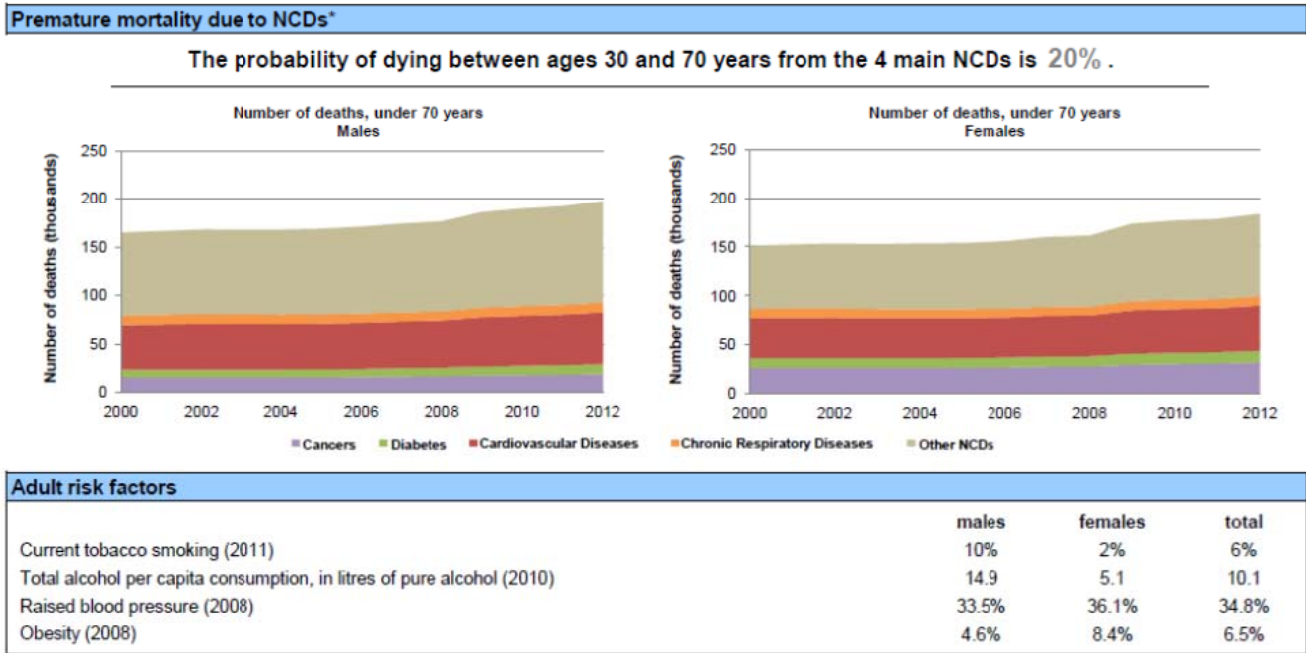

\section{National systems response to NCDs}

Has an operational NCD uni/branch or department within the Ministry of Health, or equivalent

Has an operational multisectoral national policy, strategy or action plan that integrates several NCDs and shared risk factors

Has an operational policy, strategy or action plan to reduce the harmful use of alcohol

Has an operational policy, strategy or action plan to reduce physical inactivity and/or promote physical activity

Has an operational policy, strategy or action plan to reduce the burden of tobacco use

Has an operational policy, strategy or action plan to reduce unhealthy diet and/or promote healthy diets

Has evidence-based national guidelines/protocols/standards for the management of major NCDs through a primary care approach

Has an NCD surveillance and monitoring system in place to enable reporting against the nine global NCD targets

Has a national, population-based cancer registry

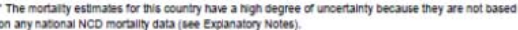

World Health Organization - Noncommunicable Diseases (NCD) Country Profiles, 2014.

Figure 2. statistics of NCDs in Nigeria and their risk factors.

\section{Determinants of health involved in NCDs}

Determinants of health are factors, which combine together to affect health of individuals and communities. They include the social, economic, physical and the individual's characteristics and behaviors. Other determinants include education, income, social status, genetics, health services, culture etc. social status and income is the single most important determinant of health as health improves with higher income and status. 
South American Journal of Public Health

Volume 4, Issue 1, 2016

Many of the determinants cannot be directly controlled by the individuals, e.g. higher income and social status gives an individual better chances of acquiring education and vice versa and it leads to more health literacy and better health. People in employment have better health especially those who have control over their working conditions. Thus they are likely to be in healthy workplaces, safe houses with clean air. In like manner, support from families, communities especially those with acceptable culture promote health. This affects personal behavior and beliefs, which can help in adoption of healthy lifestyle like avoidance of smoking, alcohol and use of health services and healthy seeking behavior. Other determinants with their effects are as shown in the figure below.

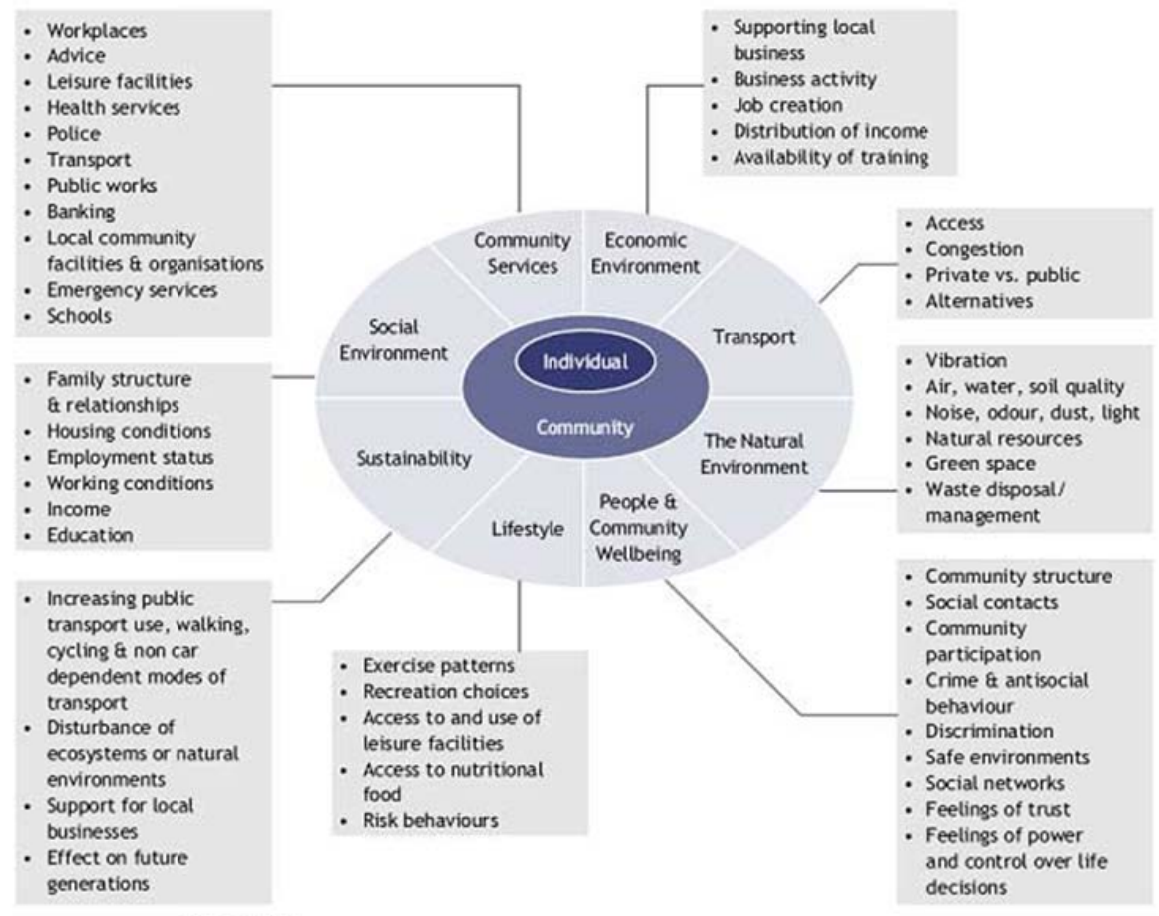

Figure 3. Determinants of Health 


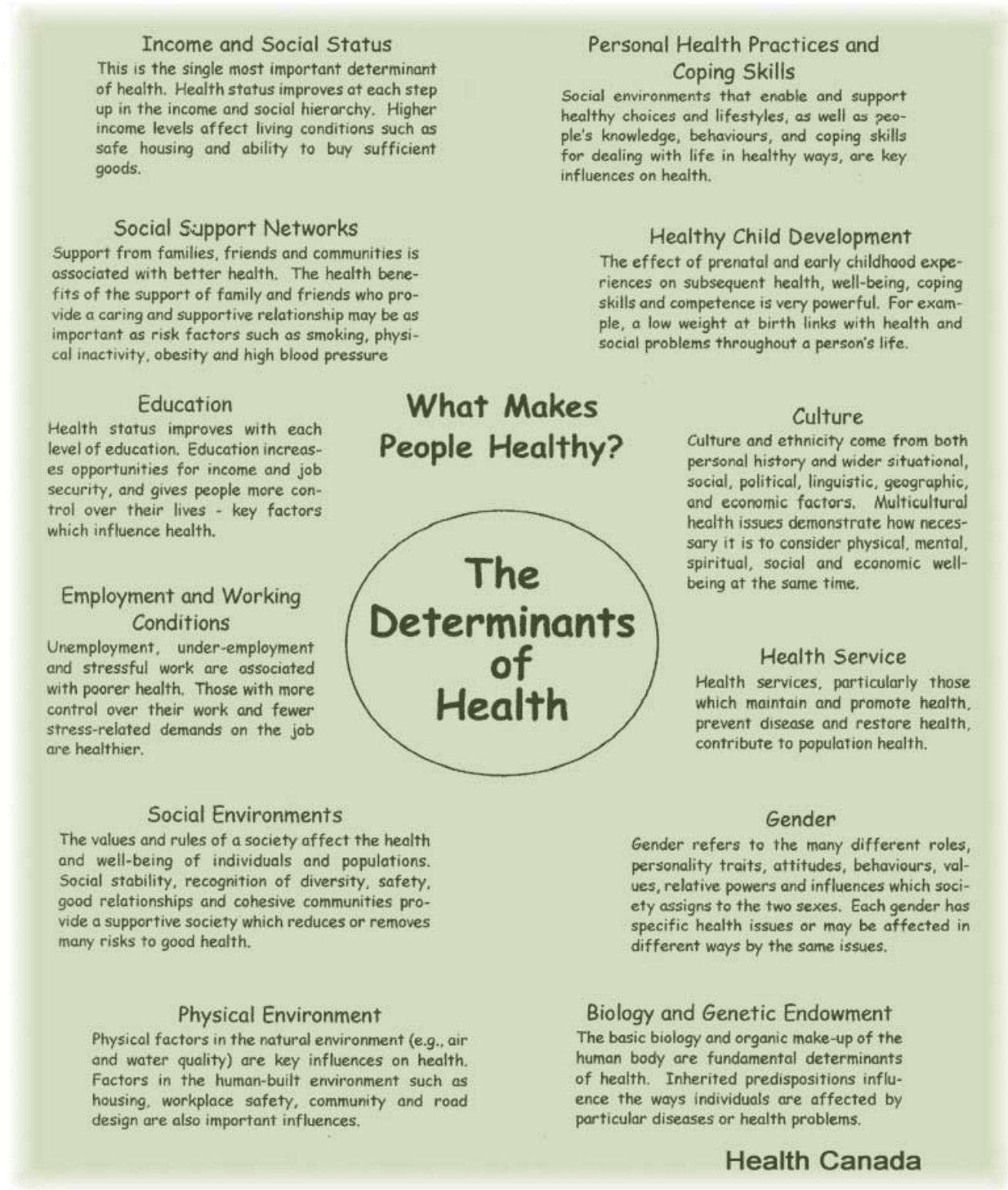

Figure 4. Details of various determinants of health.

\section{Health promotion strategies}

Health promotion is directed towards action on the determinants of health. It is aimed at effective and active public participation. It combines diverse but complementary approaches including communication, education, legislation, fiscal measures, organizational and community changes, community development and spontaneous local activities against health hazards. Government both at local and national levels has a role to play. They include health education, environmental modification, nutritional interventions and lifestyle and behavioral changes.

Health education: This is designed to improve health literacy through communication to improve knowledge and develop life skills. Health education programs and activities are used interchangeably with social marketing, mass communications, behavior modification, in-service training, patient education and some forms of health counselling. Health education on the risk factors of NCDs and how to improve the health determinants should be advocated. This includes 
information on health consequences of tobacco use, alcohol, unhealthy diet, and lack of exercise among others. This can be done through mass media campaigns, media adverts, radio phone-in programs, bulk SMS and other social networks. This also may be done by the mobilization of communities through advocacy to community leaders and stakeholders and community sensitization meetings. In addition, public campaigns and social marketing initiatives to educate and encourage consumers about healthy dietary habits should be conducted from the states down to the communities using their cultural methods. The benefits of physical activity and various methods of undertaking them should be taught. Campaigns should be linked to supporting actions across the community and within specific settings for maximum benefit and impact.

Policies on health education programs for NCDs should be formulated as part of school curriculum both in primary, secondary and tertiary institutions to form a continuum from childhood to adulthood.

Environmental modifications: Environment is the physical, chemical and biological factors external to the human host, as well as those factors impacting related behaviors. Environmental factors include the modifiable parts like pollution of air, water, or soil with chemical or biological agents, UV and ionizing radiation, noise, electromagnetic fields, occupational risks, built environments, including housing, land use patterns, roads etc. and the non-modifiable parts. Reduction of occupational risks should be considered primary to establishment of any workplace.

Government should legislate for $100 \%$ tobacco smoke-free environments in all indoor workplaces, public transport, indoor public places and other public places. Policy measures on creation of health promoting environments should be implemented prior to licensing of any establishment.

Nutritional interventions: Food is essential for life. Nutritional interventions early in life through promotion and support of exclusive breastfeeding for the first six months of life, continued breastfeeding until two years old and beyond and adequate and timely complementary feeding should be advocated. There should be implementation of WHO recommendations on the marketing of foods and non-alcoholic beverages to children, including mechanisms for monitoring. Guidelines should be developed with policy measures that engage different relevant sectors, such as food producers and processors and other relevant commercial operators, to reduce level of salt or sodium in pre-packaged foods, eliminate completely saturated fatty acids in food supply replacing them with unsaturated fatty acids, reduce the content of free and added sugars in food and non-alcoholic beverages, reduce the energy density of foods in order to limit excess calorie intake and increase consumption of fruit and vegetables.

The government in collaboration with the agricultural sector should provide policies and reforms for improvement in the provision of fruits and vegetables so that even the less privileged can afford it. Policy measures should be focused at food retailers and caterers to improve the availability and acceptability of healthy food products e.g. plant foods. Promotion and provision of healthy local agricultural food products in all public institutions including schools and workplaces should be encouraged.

Lifestyle and behavioral changes: this involves health belief of an individual/community which can be improved through information and social marketing. The various risk factors and determinants of health can be improved through behavioral changes. Physical activity can be improved through engagement of all stakeholders, non-governmental organizations, government, civil society and economic operators to implement actions aimed at increasing physical activity across all age groups. Policy measures should be developed to promote physical activity in the daily living, through recreation, leisure and sport and supportive infrastructure to support active transport i.e. walking and cycling. Other measures are improved 
provision of quality physical education from infant years to tertiary level including opportunities for physical activity before, during and after the formal school day, in addition to the creation and preservation of built and natural environments which support physical activity in workplaces, schools and in the wider communities. Promotion of community involvement in fostering local actions aimed at increasing physical activity.

Government should implement comprehensive bans on tobacco advertising, promotion and sponsorship and offer help to people who want to stop using tobacco or reduce their exposure to environmental tobacco smoke. They should also regulate the contents and emissions of tobacco products and request manufacturers and importers of the products to disclose to government authorities information regarding contents. Taxes should be raised on all tobacco products to reduce consumption.

Government should provide job opportunities to be enhance the social status of the youths most importantly to improve adoption of health promotion actions through better health literacy.

\section{Conclusion and recommendations}

Prevention and control of NCDs buttresses on prevention of risk factors, which though occurs in adulthood but exposure begins in childhood and builds up throughout life. Thus both primordial and primary prevention are necessary. Addressing the determinants of Health, which directly imparts on the risk factors, is paramount and all sectors are needed to achieve it. Primary Health Care centers staff should be regularly trained on the risks, their methods of prevention and control and methods of early detection of the various NCDs.

The government should provide an NCD operational unit/department in the Federal Ministry of Health instituting an operational multi-sectorial policy, strategies and action plans that integrates several NCDs and their shared risk factors. Also operational policy, strategy and action plan to reduce harmful use of alcohol, reduce tobacco smoking, promote physical activity, and reduce unhealthy diet while promoting healthy dietary habits should be implemented. Establishment and strengthening of NCDs surveillance systems with its associated risk factors should be conducted every 2 years in the State with standardization of the collection of data on disease incidence, risk factors and mortality by cause. A National Population based Cancer Registry should be opened.

\section{References}

[1]. Abdalla, S. Aboyans, V. "Years lived with disability (YLDs) for 1160 sequelae of 289 diseases and injuries 1990-2010: A systematic analysis for the Global Burden of Disease Study 2010", Lancet (2012), Vol.380, (9859), pp 2163-96.(PubMed PMID:23245607)

[2]. Decramer, M. Janssens, W. Miravitlles, M. "Chronic obstructive pulmonary disease", Lancet (2012), Vol.379, (9823), pp 1341-51.(PubMed PMID: 22314182)

[3]. Dobe, M. "Health promotion for prevention and control of NCD: Unfinished agenda”, Indian journal of Public Health (2012), Vol.56, pp180-6.(PubMed PMID: 23229208)

[4]. Follow-up to the Political Declaration of the High-level Meeting of the General Assembly on the Prevention and Control of NCDs. WHO (2013).

[5]. GBD 2013 Mortality and Causes of Death. "Global, regional, and national age-sex specific all-cause and cause-specific mortality for 240 causes of death, 1990-2013: a systematic analysis for the Global Burden of Disease Study (2013)."Lancet Vol.385 (9963), pp117-71.(PubMed PMID:25530442)

[6]. Global action plan for the prevention and control of non-communicable diseases 2013-2020.WHO (2013).

[7]. Health Reform Foundation of Nigeria. (2011)

[8]. International Diabetes Federation. November (2014).

[9]. International Diabetes Federation. (2013). p. 7. 
South American Journal of Public Health

Volume 4, Issue 1, 2016

[10]. Jemal, A. Bray, F. Center, M.M. Ferlay, J. Ward, E. Forman, D. "Global cancer statistics". CA: a Cancer Journal for Clinicians (2011), Vol.61 No.2, pp 69-90.(PubMed PMID:21296855)

[11]. Mendis, Shanthi, Puska, Pekka, Bo Norrving, "Global atlas on cardiovascular disease prevention and control (1st ed. Ed.). Geneva: WHO in collaboration with the World Heart Federation and the World Stroke Organization”.(2011).pp. 3-18.

[12]. Ottawa Charter for Health Promotion.

[13]. Shlomo Melmed, S.Kenneth, P. Polonsky, Reed Larsen and Henry M. Kronenberg, in Williams's textbook of Endocrinology (12th Ed.). Elsevier/Saunders publishers, Philadelphia (2011), pp. 1371-1435.

[14]. Vestbo, J. "Global Strategy for the Diagnosis, Management, and Prevention of Chronic Obstructive Pulmonary Disease”, Global Initiative for Chronic Obstructive Lung Disease (2013), pp. 1-7.(PubMed PMID:22878278)

[15]. Vos, T. Flaxman, A.D. Naghavi, M. Lozano, R. Michaud, C. Ezzati, M. Shibuya, K. Salomon, J.A. GBD 2013 Mortality and Causes of Death, Collaborators, "Global, regional, and national age-sex specific all-cause and cause-specific mortality for 240 causes of death, 1990-2013: a systematic analysis for the Global Burden of Disease Study 2013."Lancet(2014), Vol. 385, pp 117-171.(PubMed PMID:25530442)

[16]. WHO. February (2014).

[17]. World Cancer Report 2014. WHO (2014). pp. Chapter 1.1.

[18]. WHO global status report on NCDs. WHO (2011).

[19]. WHO NCD country profile. WHO (2014).

[20]. WHO Projections of mortality and causes of death, 2015 and 2030. WHO (2013).

[21]. 10 facts on NCDs. WHO (2013). 